Farm animal welfare - who writes the rules?

Occasional Publication No. 23 - British Society of Animal Science 1999

edited by A. J. F. Russel, C. A. Morgan, C. J. Savory, M. C. Appleby and T. L. J. Lawrence

\title{
Methods of assessing adequacy of drinker provision in group-housed pigs
}

\author{
S. P. Turner and S. A. Edwards \\ Scottish Agricultural College, Ferguson Building, Craibstone Estate, Bucksburn, Aberdeen AB21 9YA
}

\section{Introduction}

Competition for resources accounts for the greater part of animal aggression and access may be prioritized in favour of those of highest social rank. Consequently, under commercial conditions, provision of resources to a group of pigs must be adequate to allow all animals sufficient access, regardless of social status.

Although the volume of drinking water offered to growing pigs is generally not restricted, the ability of pigs to gain access to the drinking points themselves has received little attention. The current Welfare Codes have adopted caution in advising the provision of one nipple drinker per 10 growing pigs fed a dry diet. Producers have frequently extended this ratio to one drinker per 20 animals without encountering problems.

Furthermore, the advent of large straw court housing demands a reassessment of current resource provision. Extrapolating the drinker allocation of a small group of pigs to a larger group situation would be unwise.

Thus the purpose of this investigation was to describe the effects of drinker allocation and group size, in isolation and their interaction, on the drinking behaviour, aggression and performance of heavy, medium and light weight pigs.

\section{Material and methods}

Over the period January to July, 640 Large White $X$ Landrace growing pigs (start weight $36 \pm 5.0 \mathrm{~kg}$ ) were allocated to one of four treatments for 5 weeks. The experiment comprised of four replicates of a $2 \times$ 2 factorial design of two nipple drinker to pig ratios $(1: 10 v, 1: 20)$ and two group sizes $(20 v .60)$. Space allowance and feeding space per pig remained constant. The groups of 20 were selected from three smaller commercial pens, and the groups of 60 from nine pens.

The nipple drinkers were of standard commercial type (Arato, '80 Pig Drinker', Clacton-on-Sea, UK). A flow meter, which was calibrated regularly, recorded daily water use per pen. The flow rate of the individual drinkers was noted at the beginning of each replicate. Ambient temperature was recorded daily $1 \mathrm{~m}$ above floor height.

Two males and one female of each of three weight classes, heavy $(41.9$ (s.e. 0.57$) \mathrm{kg}$ ), medium ( 35.7 (s.e. $0.51) \mathrm{kg}$ ) and light ( 30.9 (s.e. 0.63$) \mathrm{kg}$ ), were selected from each pen on which to concentrate observations.

Daily water use per pen was used to calculate mean water use per pig. Time-lapse video recordings were made during the 4 th week on trial. Alternate $30 \mathrm{~min}$ blocks throughout the 24-h period were analysed using continuous sampling to describe the frequency and duration of drinking bouts. The number of fresh cuts, scratches and abrasions of the focal animals were counted, by a single observer, at 3 days post mixing, and at the end of the 1st and subsequent weeks, up to week 4 .

Average daily gain (ADG) was calculated from liveweight measurements made on the day of mixing and at the end of weeks 2 and 4. Average daily food intake (ADFI) and the food conversion ratio (FCR) were calculated on a per pen basis.

All values were corrected for the number of animals on trial. The influence of replicate was examined in each case. All parameters were analysed using a randomized block analysis of variance or two-way ANOVA.

\section{Results}

Considering all members of the pen, individuals in larger groups used more water than those in smaller groups, irrespective of drinker allocation (5.04 v. 3.66 (s.e.d. 0.230$) 1$ per pig per day, $P<0.001$ ). Heavy, medium and light weight pigs visited the drinkers a similar number of times $(1.16,1.31$ and 1.14 (s.e.d. $0 \cdot 161$ ) visits per pig per $h$, respectively), performed drinking bouts of similar duration $(26.6,23.5$ and 24.9 (s.e.d. 2.55) s per visit), and consequently spent a similar amount of time drinking per day $(722,762$ 


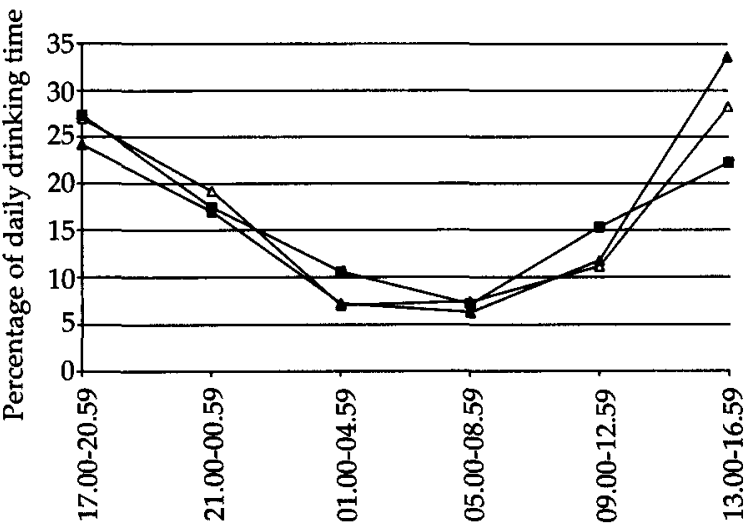

Time in 24-h period

Figure 1 Percentage of daily drinking time occuring during 4- $h$ blocks for each weight class when treatment was pooled: $\triangle-$ heavy; $\rightarrow-$ medium; $\rightarrow$ light.

and 701 (s.e.d. 90.6) s per pig per day). Weight class and treatment did not interact to significantly affect these parameters.

The 24-h period of video analysis of each pen was divided into six blocks of $4 \mathrm{~h}$. Plotting the percentage of daily drinking time occurring during each block revealed no effect of treatment on the diurnal pattern of drinking in any weight class (Figure 1).

Treatment, in isolation, or in interaction with weight class, did not significantly affect the lesion score during either the whole period, or at 3 days post mixing alone $(11.1,9.3,11.0$ and 12.2 (s.e.d. 1.36) lesions per pig for pooled focal pigs in 60 pigs three drinkers, 20 pigs - one drinker, 60 pigs - six drinkers and 20 pigs - two drinkers respectively over the whole trial period).

The mean ADG, ADFI, and FCR of the pen was not affected by treatment. The interaction between treatment and focal pig weight class did not affect
ADG. Pigs beginning the experiment with a lower inter-quartile start weight gained more slowly in a group of 60 with three drinkers (Table 1). This pattern was repeated by the pigs with an upper inter-quartile start weight and the interaction between start weight and treatment was not significant.

\section{Discussion}

The drinking behaviour of the lightest animals in the pen closely matched that of the heaviest, indicating that weight (and possibly, by inference, rank) was not correlated with drinker access. Alternatively, the resource was not sufficiently limited to encourage the necessary degree of competition. This may account for the similarity in diurnal use of the drinkers for heavy, medium and light weight animals in each treatment. From the results of various workers (Gonyou and Stricklin (1981), Stricklin and Gonyou (1981) in beef cattle and Morrow and Walker (1994) in pigs), sub-optimal feeder allocation caused subordinate animals to make frequent visits to the feeder at night. The lack of an interaction between weight and treatment on lesion score further suggests that the intensity of competition was similar in each treatment.

The lower ADG of the upper-interquartile start weight animals in a group of 60 pigs with three drinkers indicated that the low weight pigs were not being selected against specifically by this treatment. The absence of an interaction between weight and treatment on performance in our trial, suggests that the competition pressure was not sufficient to highlight a weight effect on resource access, should one exist.

Preferential access of heavier animals to resources was not observed, and, moreover, light-weight animals were not selectively penalized with respect to their drinking behaviour, the aggression they received, or their ADG in any treatment. It is probable that the restrictions imposed by each of the

Table 1 Average daily gain $(\mathrm{kg})$ of the pen, focal pigs and pigs of lower and upper inter-quartile start weight in the four combinations of group size and drinker allocation

\begin{tabular}{|c|c|c|c|c|c|c|}
\hline & \multicolumn{4}{|c|}{ Treatment } & \multirow[b]{2}{*}{ s.e.d. } & \multirow{2}{*}{$\begin{array}{c}\text { Significancet of } \\
\text { drinkers } X \\
\text { group size }\end{array}$} \\
\hline & $\begin{array}{c}60 \text { pigs, } \\
3 \text { drinkers }\end{array}$ & $\begin{array}{l}20 \text { pigs, } \\
1 \text { drinker }\end{array}$ & $\begin{array}{l}60 \text { pigs, } \\
6 \text { drinkers }\end{array}$ & $\begin{array}{c}20 \text { pigs, } \\
2 \text { drinkers }\end{array}$ & & \\
\hline Pen mean & 0.69 & 0.68 & 0.66 & 0.67 & $0 \cdot 029$ & \\
\hline Pooled focal pigs & $0 \cdot 70$ & $0 \cdot 70$ & $0 \cdot 65$ & $0 \cdot 67$ & 0.034 & \\
\hline Lower quartile & 0.53 & 0.68 & 0.68 & 0.59 & 0.059 & * \\
\hline Upper quartile & 0.63 & 0.80 & $0 \cdot 70$ & 0.64 & $0 \cdot 046$ & $* *$ \\
\hline
\end{tabular}

+ Drinker effects and group size effects were not significant $(P>0-05)$. 
treatments was not adequate to cause an affect of weight on these parameters. Thus, in a large group of 60 pigs, with one drinker per 20 animals, the welfare and performance of the lightest individuals did not suffer.

\section{Acknowledgements}

SPT gratefully acknowledges the support of the Cruden Foundation. SAC receives financial support from SOAEFD. The authors wish to thank all of the staff at Tillycorthie Farm for their assistance.

\section{References}

Gonyou, H. W. and Stricklin, W. R. 1981. Eating behavior of beef cattle groups fed from a single stall or trough. Applied Animal Ethology 7: 123-133.

Morrow, A. T. S. and Walker, N. 1994. Effects of number and siting of single space feeders on performance and feeding behaviour of growing pigs. Journal of Agricultural Science, Cambridge 122: 465-470.

Stricklin, W. R. and Gonyou, H. W. 1981. Dominance and eating behavior of beef cattle fed from a single stall. Applied Animal Ethology 7: 135-140. 